\title{
Microstimulation of the Monkey Superior Colliculus Induces Pupil Dilation Without Evoking Saccades
}

\author{
Chin-An Wang, Susan E. Boehnke, Brian J. White, and Douglas P. Munoz \\ Centre for Neuroscience Studies, Queen's University, Kingston, Ontario K7L 3N6, Canada
}

The orienting reflex is initiated by a salient stimulus and facilitates quick, appropriate action. It involves a rapid shift of the eyes, head, and attention and other physiological responses such as changes in heart rate and transient pupil dilation. The SC is a critical structure in the midbrain that selects incoming stimuli based on saliency and relevance to coordinate orienting behaviors, particularly gaze shifts, but its causal role in pupil dilation remains poorly understood in mammals. Here, we examined the role of the primate SC in the control of pupil dynamics. While requiring monkeys to keep their gaze fixed, we delivered weak electrical microstimulation to the SC, so that saccadic eye movements were not evoked. Pupil size increased transiently after microstimulation of the intermediate SC layers (SCi) and the size of evoked pupil dilation was larger on a dim versus bright background. In contrast, microstimulation of the superficial SC layers did not cause pupil dilation. Thus, the SCi is directly involved not only in shifts of gaze and attention, but also in pupil dilation as part of the orienting reflex, and the function of pupil dilation may be related to increasing visual sensitivity. The shared neural mechanisms suggest that pupil dilation may be associated with covert attention.

\section{Introduction}

When a salient event occurs in the environment, the body generates a complex orienting reflex that involves behavioral and physiological changes such as rapid shifts of gaze and attention and changes in heart rate (Hess et al., 1946; Sokolov, 1963a; Boehnke and Munoz, 2008). The purpose of this reflex is to heighten perception to efficiently discern what is happening and ready the body for whatever action is required (Sokolov, 1963b).

Pupil dilation has long been characterized as one component of the orienting reflex (Sokolov, 1963a; Lynn, 1966). A considerable body of results has shown that pupil size increases transiently after the presentation of salient stimuli (Stelmack and Siddle, 1982; Bala and Takahashi, 2000; Netser et al., 2010), and the function of pupil dilation is thought to increase the sensitivity of sense organs (Lynn, 1966), although supporting evidence is less established (Nieuwenhuis et al., 2011).

The midbrain SC (or the optic tectum in nonmammalian vertebrates) is hypothesized to coordinate the orienting reflex (Boehnke and Munoz, 2008), because it receives convergent in-

\footnotetext{
Received Nov. 1, 2011; revised Dec. 22, 2011; accepted Jan. 14, 2012.

Author contributions: C.-A.W. and D.P.M. designed research; C.-A.W. performed research; C.-A.W., S.E.B., and B.J.W. contributed unpublished reagents/analytic tools; C.-A.W. analyzed data; C.-A.W., S.E.B., B.J.W., and D.P.M. wrote the paper.

This work was supported by Canadian Institutes of Health Research Grant MOP-77734 and a Defense Advanced Research Projects Agency Neovision2 Program grant. D.P.M. was supported by the Canada Research Chair Program. We thank Ann Lablans, Donald Brien, Sean Hickman, Diane Fleming, and Mike Lewis for outstanding technical assistance and members of the Munoz laboratory for comments on an earlier version of the manuscript. The views and conclusions contained in this document are those of the authors and should not be interpreted as representing the official policies, either expressly or implied, of the Defense Advanced Research Projects Agency or the U.S. Government.

Correspondence should be addressed to Douglas P. Munoz, Centre for Neuroscience Studies, Queen's University, Room 234, Botterell Hall, 18 Stuart Street, Kingston, ON K7L 3N6, Canada. E-mail: doug.munoz@biomed.queensu.ca.

DOI:10.1523/JNEUROSCI.5512-11.2012

Copyright $\odot 2012$ the authors $\quad 0270-6474 / 12 / 323629-08 \$ 15.00 / 0$
}

puts from multiple cortical and subcortical brain areas and projects not only back to most of the cortex, but also directly to the brainstem and spinal cord to execute orienting movements. The SC is conserved phylogenetically across a range of species (Hall and Moschovakis, 2003), and it is organized into a retinotopic map of contralateral visual space with functionally differentiated layers. The superficial layers (SCs) receive inputs from early visual areas including the retina, whereas the intermediate layers (SCi) receive inputs from multisensory, motor, and cognitive related areas (Sparks, 1986; White and Munoz, 2011). Saccaderelated neurons in the SCi project to the premotor circuitry in the brainstem reticular formation and the spinal cord to initiate movements of the eyes and head (Moschovakis et al., 1988b; Rodgers et al., 2006). Electrical microstimulation in the SCi in monkeys induces saccades (Robinson, 1972) and biases attention (Kustov and Robinson, 1996; Cavanaugh and Wurtz, 2004; Müller et al., 2005) toward spatially aligned locations in the SC retinotopic map. Recently, it was shown that microstimulation of the deep layers of the optic tectum in anesthetized barn owls evokes pupil dilation (Netser et al., 2010), which importantly extends the role of the SC to other components of the orienting reflex, although this has yet to be established in awake behaving monkeys.

Although the SC has a well known causal role in the generation of automatic gaze shifts to salient stimuli (Mohler and Wurtz, 1977; Hikosaka and Wurtz, 1983; Schiller et al., 1987), its role in pupil dilation is poorly understood. Here, we examine the effect of subthreshold microstimulation (i.e., saccades not evoked) of the SC of two behaving rhesus monkeys on pupil dynamics. Our results demonstrate that the pupils dilated transiently after stimulation of the SCi, but not of the SCs, and we found this to be particularly pronounced in low levels of illumination, suggesting that the function of pupil dilation mediated by the SCi may be related to enhancing visual sensitivity. 


\section{Materials and Methods}

Animal preparation and equipment. Experiments were performed on two male rhesus monkeys (Macaca mulatta; 11 and $12 \mathrm{~kg}$ ). The protocols used in this study were approved by Queen's University Animal Care Committee in accordance with the Canadian Council on Animal Care policies on the use of laboratory animals. The methods of surgical procedures, techniques for extracellular neuronal recording, and data collection have been described in detail previously (Marino et al., 2008). Eye position was measured by the scleral search coil technique (Robinson, 1963), and horizontal and vertical eye positions were digitized at 1000 Hz. Pupil diameter was measured by using a video-based eye tracker (Eyelink-II, SR Research) at a rate of $500 \mathrm{~Hz}$, and pupil size resolution was $\sim 0.01 \mathrm{~mm}$. Pupil-size data can be distorted by eye movements, because the size of the pupil depends on the subject's gaze angle in a video-based eye tracker. To maintain an accurate measure of pupil size before, during, and after microstimulation, monkeys were required to maintain visual fixation on a point at the center of the screen throughout the trial. Stimulus presentation and data acquisition were controlled by a UNIX-based real-time data control system (REX) (Hays et al., 1982). Spikes, eye position, and pupil diameter were recorded in a multichannel data acquisition system (Plexon). Stimuli were presented on a CRT monitor at a screen resolution of $1024 \times 768$ pixels $(75 \mathrm{~Hz}$ noninterlaced), subtending a viewing angle of $54 \times 44^{\circ}$.

Procedure, SC recording, and stimulation. Monkeys were seated in a primate chair with their heads restrained facing the video monitor. Once the SC had been located by single neuron recording and the visual response fields were mapped by using a rapid visual stimulation procedure (White et al., 2009), monkeys performed a delayed saccade task to characterize the types of neurons. Each trial started with fixation of a central fixation spot $\left(0.5^{\circ}\right.$ diameter, $\left.30 \mathrm{~cd} / \mathrm{m}^{2}\right)$ against a black background for $500-800 \mathrm{~ms}$, and then a target stimulus $\left(0.5^{\circ}\right.$ diameter, $\left.30 \mathrm{~cd} / \mathrm{m}^{2}\right)$ appeared in the response field of the neuron. After a delay $(500-800 \mathrm{~ms})$, the fixation spot was removed and the monkey was required to generate a saccade toward the target. Because target presentation was temporally dissociated from the saccade, the visual and motor components of the discharge were isolated and easily distinguished. Spike rasters were generated in real time to confirm the presence or absence of visual and motor activity.

We lowered tungsten microelectrodes (impedance 0.1-1 M $\Omega$; Frederick Haer) to the SC. The dorsal surface was easily identified by multiunit visual activity. The SC was defined as the depth at which neuronal activity clearly indicated the presence of visual activity after the appearance of each target ( $>50$ spike/s within $100 \mathrm{~ms}$ after the target appearance) and no saccade-related activity (Cynader and Berman, 1972; Goldberg and Wurtz, 1972). Then, we advanced the electrode further to determine the depth of the SCi, which was defined as the point at which neurons had pronounced increases in discharge related to the initiation of saccades (>80 spikes/s $\pm 5 \mathrm{~ms}$ on saccade onset) (Schiller and Koerner, 1971; Wurtz and Goldberg, 1971; Sparks, 1978). Once the SCi was confirmed, the SCi was microstimulated $(300 \mathrm{~Hz}$ pulse train for $100 \mathrm{~ms}$ with alternating $0.3 \mathrm{~ms}$ anode plus $0.3 \mathrm{~ms}$ cathode pulses), and threshold for saccades was determined when the stimulation current in the SCi evoked saccades $50 \%$ of the time (range 5-50 $\mu \mathrm{A}$ ). Generation of a saccade reduced the accuracy of pupil measurement because of the change in viewing angle, and it confounded our test of the role of the SC in pupil dilation, because any observed dilation could be triggered by the saccadic eye movement itself, rather than SC microstimulation per se. To get an accurate estimation of pupil size and avoid an influence of saccadic eye movements, we used $50-70 \%$ of the saccade threshold current determined separately for each site in the SCi in a given session so that saccadic eye movements were not evoked. The subthreshold current derived for the SCi was used for microstimulation of both the SCs and SCi. The confirmation of stimulation sites in the SCi was always made by evoking a saccade toward the estimated response field with suprathreshold stimulation. For SC stimulation, we simply raised the electrode to the previously marked depth of the SCs and confirmed again that neurons had only visual activity in the absence of any motor response. Localization of the SCs was further confirmed by the failure to evoke saccades with the suprathreshold microstimulation parameters used in the SCi. The optimal locations of the response fields of SCi neurons were in close agreement with the vector of eye movement elicited with suprathreshold SCi stimulation, so we used the optimal locations of the response fields for analysis of spatial effects. Although the experiment used subthreshold microstimulation, we microstimulated four sites with the suprathreshold current from two monkeys to examine whether "suprathreshold" and "subthreshold" microstimulation resulted in similar pupil dilation. We microstimulated sites in the rostral SC to evoke very small vector saccades (the optimal response field: $x=-0.6^{\circ}, y=-1.4^{\circ}$ and $x=0.8^{\circ}, y=$ $0.4^{\circ}$ from two monkeys) to test the hypothesis without diminishing the accuracy of pupil size measurement before, during, and after microstimulation. As observed with subthreshold stimulation, there was an initial transient dilation of pupil, followed by a sustained increase in pupil size (phasic epoch: $t_{(3)}=2.36, p=0.09$; sustained epoch: $t_{(3)}=$ $2.65, p=0.07)$.

Behavioral task. Monkeys were trained to perform a simple fixation task. They had to maintain gaze within $1.5^{\circ}$ of a fixation spot $\left(0.5^{\circ}\right.$ diameter, $30 \mathrm{~cd} / \mathrm{m}^{2}$ ) at the center of the screen on a background for 3-4 s to obtain a liquid reward. After the monkey maintained fixation for $1.5-2 \mathrm{~s}$, a train of stimulation pulses was delivered $(100 \mathrm{~ms}, 300 \mathrm{~Hz}, 50-70 \%$ saccade threshold) on $50 \%$ of the trials, then monkeys had to maintain fixation for another 1.5-2 s regardless of microstimulation. Two arbitrarily selected values of background luminance were implemented (25 or $35 \mathrm{~cd} / \mathrm{m}^{2}$; referred as a relatively dim or bright condition) to examine whether the size of electrically evoked pupil dilation changed as a function of background luminance. All conditions were randomly interleaved. Microstimulation was delivered to 28 sites in the SCi (18 in monkey A and 10 in monkey B) and 10 sites in the SCs (4 in monkey A and 6 in monkey B). There were at least 30 correct trials in all conditions.

Data analysis. Trials with blinks during the required period of fixation (3-4 s) were excluded from analysis. For the majority of stimulation sites, both pupils were recorded (SCi: 23/28 sites; SCs: 10/10 sites), and pupil diameter of the right eye was used mainly for data analysis. To normalize pupil diameter, for each trial a baseline pupil value was determined by averaging pupil size during the epoch $500 \mathrm{~ms}$ before the onset of electrical stimulation. Pupil values were subtracted from this baseline value. Similar procedures have been used previously (Bala and Takahashi, 2000; Moresi et al., 2008).We used the method similar to Netser et al. (2010) to quantify the magnitude of pupil dilation. The pupil dilation value was defined as the average value of the normalized pupil diameter during two selected time windows that captured the pupillary changes evoked by microstimulation (Fig. $1 B, C$ ), a phasic epoch $(150-450 \mathrm{~ms}$ after the stimulation onset), and a sustained epoch (600-1200 ms after the stimulation onset). To simplify the data, we contrasted the normalized pupil diameter values between the stimulation versus nostimulation conditions directly (see Fig. 5; this calculation was also applied to Figs. $3 C, D, 4)$. To calculate the latency of pupil dilation, we used the average pupil value in a sliding $10 \mathrm{~ms}$ window starting from stimulation onset to $1500 \mathrm{~ms}$ poststimulation and compared the average stimulation versus no-stimulation pupil value with a $t$ test. Dilation onset latency was defined as the earliest point in which the stimulation pupil size statistically exceeded the no-stimulation pupil size $(p<0.05)$ and remained so for at least $10 \mathrm{~ms}$.

\section{Results}

Microstimulation was delivered to 28 sites in the SCi (18 in monkey $\mathrm{A}$ and 10 in monkey B). The optimal locations of the response fields determined by neuronal activity recorded at these sites ranged between 2 and $35^{\circ}$ eccentricity and are transformed and plotted in Figure $1 A$ in SC coordinates (Van Gisbergen et al., 1987). Figure $1, B$ and $C$, shows the effects of SCi microstimulation on pupil diameter at an example stimulation site in monkeys A and B, respectively (collapsed across background luminance). Microstimulation resulted in an initial transient increase in pupil size (highlighted in the leftmost shaded region in Fig. $1 B, C$, from 150 to $450 \mathrm{~ms}$ poststimulation), followed by a relative sustained increase in pupil size (highlighted in the shaded region in Fig. 
A SCi stimulation sites

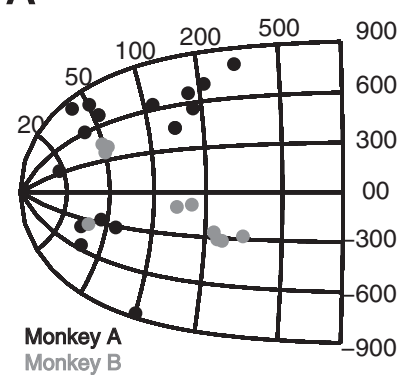

D SCs stimulation sites

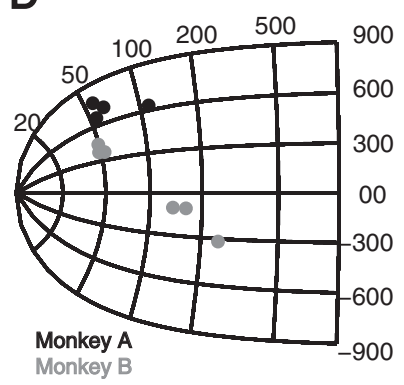

B Monkey A (SCi example site)

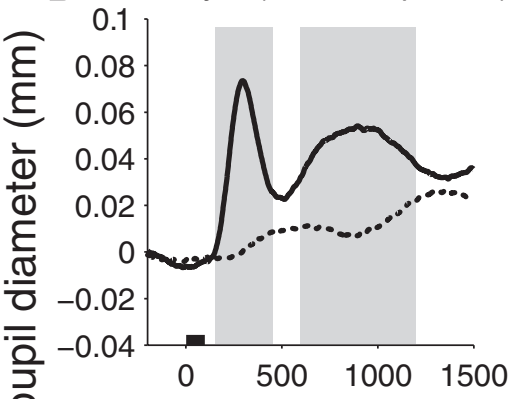

응 $E$

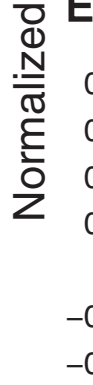

Monkey A (SCs example site)

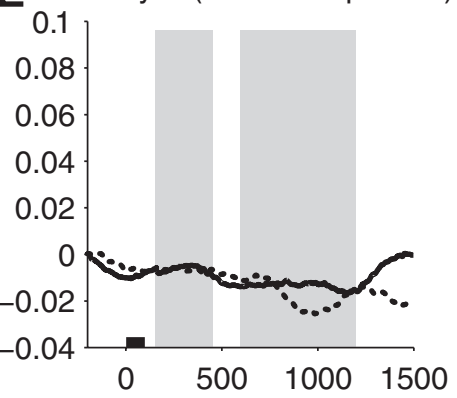

C Monkey B (SCi example site)

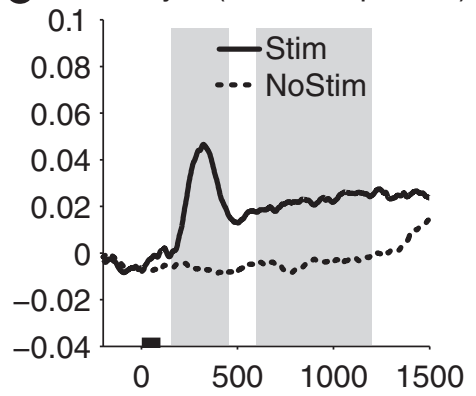

F Monkey B (SCs example site)

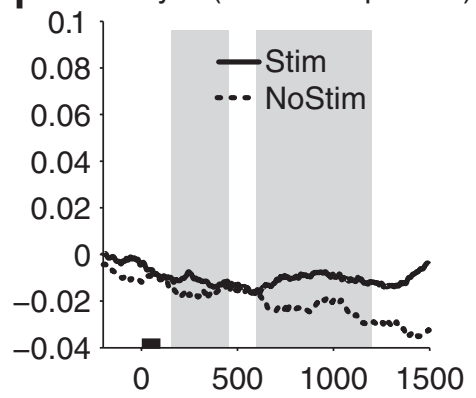

Time from stimulation onset (ms)

Figure 1. The sites and effects of microstimulation of the SCi and the SC layers in two monkeys. $\boldsymbol{A}, \boldsymbol{D}, \mathrm{SCi}(\boldsymbol{A})$ and SCS $(\boldsymbol{D})$ stimulation sites transformed into SC coordinates (Van Gisbergen et al., 1987). Black and gray circles indicate data in monkeys $A$ and $B$, respectively. $\boldsymbol{B}, \boldsymbol{C}$, Examples of the effects of SCi microstimulation on the normalized pupil diameter in monkey $A(\boldsymbol{B})$ and monkey $B$ (C). $\boldsymbol{E}, \boldsymbol{F}$, Examples of the effects of $S(S$ microstimulation in monkey $A(\boldsymbol{E})$ and monkey $B(\boldsymbol{F})$. In $\boldsymbol{B}, \boldsymbol{C}, \boldsymbol{E}$, and $\boldsymbol{F}$, the solid and dashed lines indicate the microstimulation and no-stimulation trials, respectively, and black bars indicate the time line of microstimulation. The 150-450 and 600-1200 ms epochs after the stimulation onset are shaded in gray. Stim, Stimulation; NoStim, no stimulation.

$1 B, C$, from 600 to 1200 ms poststimulation). These differences were highly significant (monkey A: phasic epoch, $t_{(197)}=8.62$, $p<0.0001$, sustained epoch, $t_{(197)}=5.36, p<0.0001$; monkey B: phasic epoch, $t_{(130)}=7.49, p<0.0001$, sustained epoch, $t_{(130)}=$ $5.66, p<0.0001)$. The latency of the onset of the stimulationinduced dilation was 156 and $183 \mathrm{~ms}$ after stimulation onset for monkey A and monkey B at the example stimulation sites, respectively (monkey A: $t_{(197)}=2.02, \mathrm{SD}=20.49, p<0.05$; monkey B: $\left.t_{(130)}=2.07, \mathrm{SD}=14.63, p<0.05\right)$. Because a variety of factors related to the trial presentation could affect the pupil diameter, the pupil could be undergoing constriction or dilation (or even relatively static situation) at the time of stimulation/nonstimulation (e.g., there was a clear increase in pupil size over time even in the nonstimulation condition in Fig. $1 B$ ). Therefore, examining the difference between the stimulation and nonstimulation conditions is critical to understanding the data. If microstimulation has no effect, then the pupil traces on the stimulation conditions should be similar to the nonstimulation conditions. However, SCi microstimulation transiently increased pupil size consistently.

Figure $2 A$ summarizes the effects of SCi microstimulation on pupil dynamics collapsed across monkeys and stimulation sites. SCi microstimulation caused a significant increase in pupil size (150-450 ms epoch: $t_{(27)}=7.5, p<0.0001 ; 600-1200$ ms epoch: $\left.t_{(27)}=4.99, p<0.0001\right)$. Almost all stimulation sites in our example showed numerically larger pupil dilation on the stimulation condition than on the no-stimulation condition (Fig. $2 \mathrm{~B}$ : 150-450 ms epoch, 27/28 stimulation sites, 96\%; Fig. 2C: 600$1200 \mathrm{~ms}$ epoch, 24/28 stimulation sites, $86 \%$ ). Significant differences ( $t$ test, $p<0.05$ ) were obtained for $54 \%$ of the stimulation sites ( $15 / 28$ sites) for the $150-450 \mathrm{~ms}$ epoch and $18 \%$ (5/28 sites) for the 600-1200 ms epoch. Although the size of pupil dilation was significant larger with microstimulation on the sustained epoch, its statistical values were less reliable (Fig. 2C). Moreover, pupil traces on the sustained epoch in the stimulation condition mirrored traces without microstimulation (Fig. $2 A$ ). The differences of pupil size with/without microstimulation on the sustained epoch could be attributed to an additive effect from an initial phasic pupillary change. Therefore, the reliability of the sustained response can be questioned. Caution is needed in explaining these data.

The same subthreshold current delivered to the SCi was also used to stimulate the SCs (see Materials and Methods). We stimulated 10 sites in the SCs ( 4 in monkey A and 6 in monkey B; $\geq 30$ correct trials in all conditions) with eccentricities of the response fields ranging between 5 and $25^{\circ}$ (Fig. $1 D$ ). Pupil dynamics for one example site in monkey A and B (plotted in Fig. $1 E, F$ ) show that subthreshold microstimulation of the SCs layers did not cause pupil dilation (monkey A: phasic epoch, $t_{(109)}=0.03$, sustained epoch, $t_{(109)}=0.7$; monkey B: phasic epoch, $t_{(96)}=0.33$, sustained epoch, $t_{(96)}=1.29$; all $p>0.19$ ). The combined data of two monkeys from all stimulation sites in the SCs consistently revealed that the pupil did not respond to microstimulation (Fig. 2D) (150-450 ms epoch: $t_{(9)}=0.25, p=0.81 ; 600-1200 \mathrm{~ms}$ epoch: $\left.t_{(9)}=0.07, p=0.94\right)$. No reliably greater pupil dilation was found on the stimulation versus no-stimulation conditions for both epochs across stimulation sites (Fig. $2 E, F$ ), and the differences did not reach statistical significance $(t$ test, $p<0.05)$.

If the effect of stimulation-evoked pupil dilation is related to enhanced visual sensitivity, then the amount of dilation should be constrained so it does not surpass the natural pupil size required to maximize visual acuity for the given state of back- 

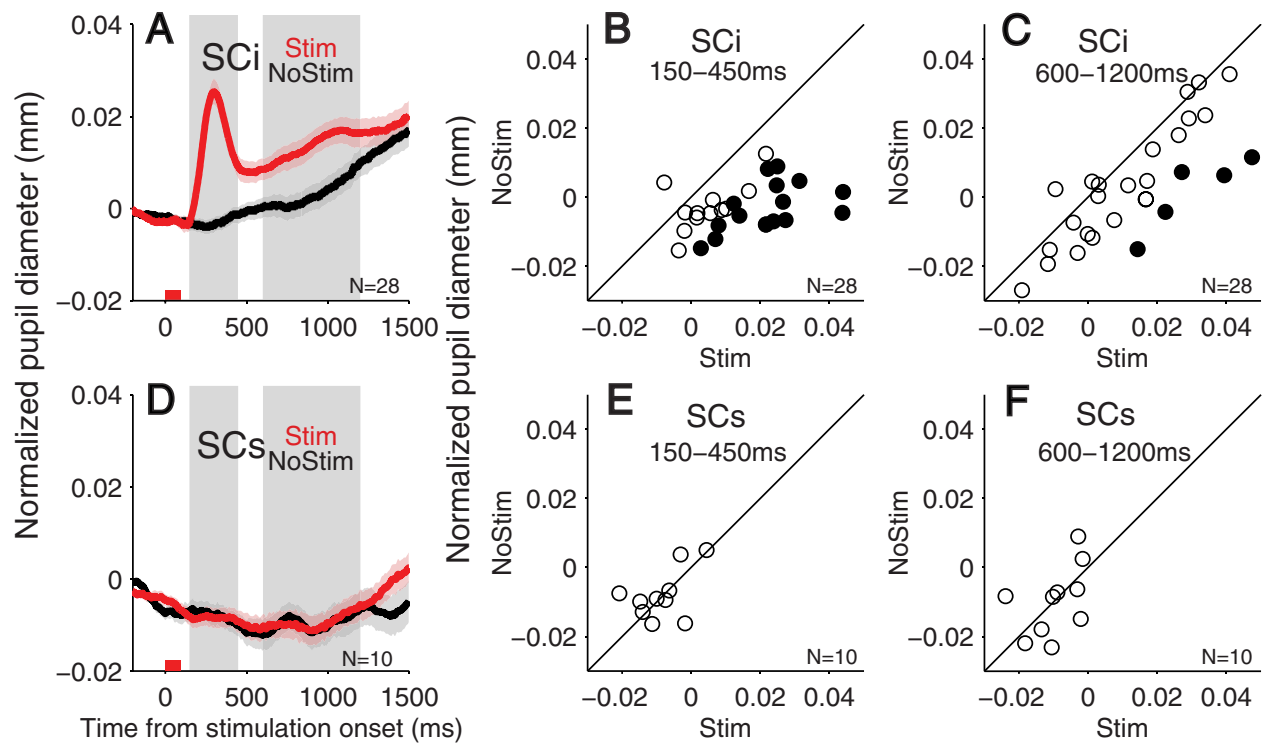

Normalized pupil diameter $(\mathrm{mm})$

Figure 2. Summary of microstimulation effects in the SCi and the SCs collapsed across monkeys and stimulation sites. $A, \boldsymbol{D}$, Pupil dynamics after SCi (A) and SC (D) microstimulation. Red and black lines indicate the microstimulation and no-stimulation trials, respectively. Red bars indicate the time line of microstimulation. The $150-450$ and $600-1200$ ms epochs after the stimulation onset are shaded in gray. The shaded regions and pupillary response traces represent \pm 1 SEM. $B, C, E, F$, Scatterplots of the pupil dilation values during stimulation versus no-stimulation trials in the SCi $(\boldsymbol{B}, \boldsymbol{C})$ and SCs $(\boldsymbol{E}, \boldsymbol{F})$ during the $150-450$ and $600-1200 \mathrm{~ms}$ epochs. Filled circles indicate sites with statistically significant differences $(p<0.05)$. Stim, Stimulation; NoStim, no stimulation.

ground illumination. Moreover, the natural pupil size is larger for lower levels of illumination (maximize visual resolution), so the size of evoked pupil dilation should be larger for a dimmer condition. To test whether the size of electrically evoked pupil dilation was modulated by the level of background illumination, we separated conditions of different background luminance. The absolute pupil size was larger on the dim background than on the bright background before stimulation onset (the baseline epoch in Fig. $3 A)\left(t_{(27)}=27.52, p<0.0001\right)$. Figure $3 B$ summarizes the effects of the SCi microstimulation on normalized pupil dynamics in the two different background conditions. The pupil dilated significantly on both background luminance (phasic epoch: dim, $t_{(27)}=7.32, p<0.0001$, bright, $t_{(27)}=5.55, p<0.0001$; sustained epoch: $\operatorname{dim}, t_{(27)}=5.02, p<0.0001$, bright, $t_{(27)}=2.53, p<$ $0.05)$. The latency of pupil dilation was 145 and $151 \mathrm{~ms}$ after stimulation onset in the dim and bright conditions, respectively $\left(\operatorname{dim}: t_{(27)}=2.44, \mathrm{SD}=0.001, p<0.05\right.$; bright: $t_{(27)}=2.22, \mathrm{SD}=$ $0.001, p<0.05)$. Critically, the data collapsed across stimulation sites showed that SCi microstimulation produced greater dilation of pupil during the dim compared with bright conditions (150$450 \mathrm{~ms}$ epoch in Fig. 3C: 22/28 stimulation sites, $96 \%, t_{(27)}=$ 2.71, $p<0.05 ; 600-1200$ ms epoch in Fig. 3D: 19/28 stimulation sites, $\left.86 \%, t_{(27)}=2.32, p<0.05\right)$, although the differences from each individual site were not consistently significant $(t$ test, $p<0.05)$ across all stimulation sites $(150-450 \mathrm{~ms}$ epoch: $7 / 28$ stimulation sites, 25\%; 600-1200 ms epoch: 5/28 stimulation sites, $18 \%$ ). The results are consistent with the idea that pupil dilation is associated with increasing visual acuity (Campbell and Gregory, 1960), because the dilation was greater with a dim background.

We also examined the relationships between the amount of pupil dilation and the eccentricity of the response field in degrees on the SC map. The size of pupil dilation was not modulated by the eccentricity of response field for the phasic epoch $(r=0.08$, $p=0.69$ ) (Fig. 4A), but was negatively correlated to the eccentricity of response field for the sustained epoch $(r=-0.44, p<$
0.05) (Fig. 4B). Although these results might suggest that the spatial information of SC neurons was carried through to signal pupil dilation on the sustained response, the correlation was not strong enough to be conclusive. Moreover, as stated, the validity of the sustained response still requires future investigation. The pronounced initial pupil dilation regardless of a stimulation location on the SC map may imply that the pupil responds equally to salient stimuli at different locations.

Finally, we contrasted normalized pupil dynamics (see Materials and Methods) between the pupil contralateral versus the ipsilateral to the side of microstimulation (Fig. 5A) (data were recorded from both pupils at 23 sites of SCi stimulation). SCi microstimulation induced bilateral pupil dilation. Figure 5, $B$ and $\mathrm{C}$, plots dilation values for the contralateral pupil against those of the ipsilateral pupil for each microstimulation site for the phasic and sustained epochs, respectively. There were no differences between the two pupils (phasic epoch: $t_{(22)}=0.004, p=0.96$; sustained epoch: $\left.t_{(22)}=0.33, p=0.74\right)$, indicating both pupils responded equally to stimulation from one SCi.

\section{Discussion}

We used microstimulation to examine whether the SC was involved in the control of pupil dilation. Microstimulation of the SCi, but not of the SCs, caused bilateral transiently pupil dilation under both levels of background illumination used in the current study, with the magnitude of stimulation-induced pupil dilation being more pronounced against a lower background luminance. The results extend the hypothesis that the SCi coordinates not only shifts gaze and attention (Kustov and Robinson, 1996; Cavanaugh and Wurtz, 2004; Müller et al., 2005), but also pupil dilation as one component of the orienting reflex (Boehnke and Munoz, 2008; Netser et al., 2010). Moreover, the function of evoked pupil dilation may be associated with increasing visual sensitivity. 

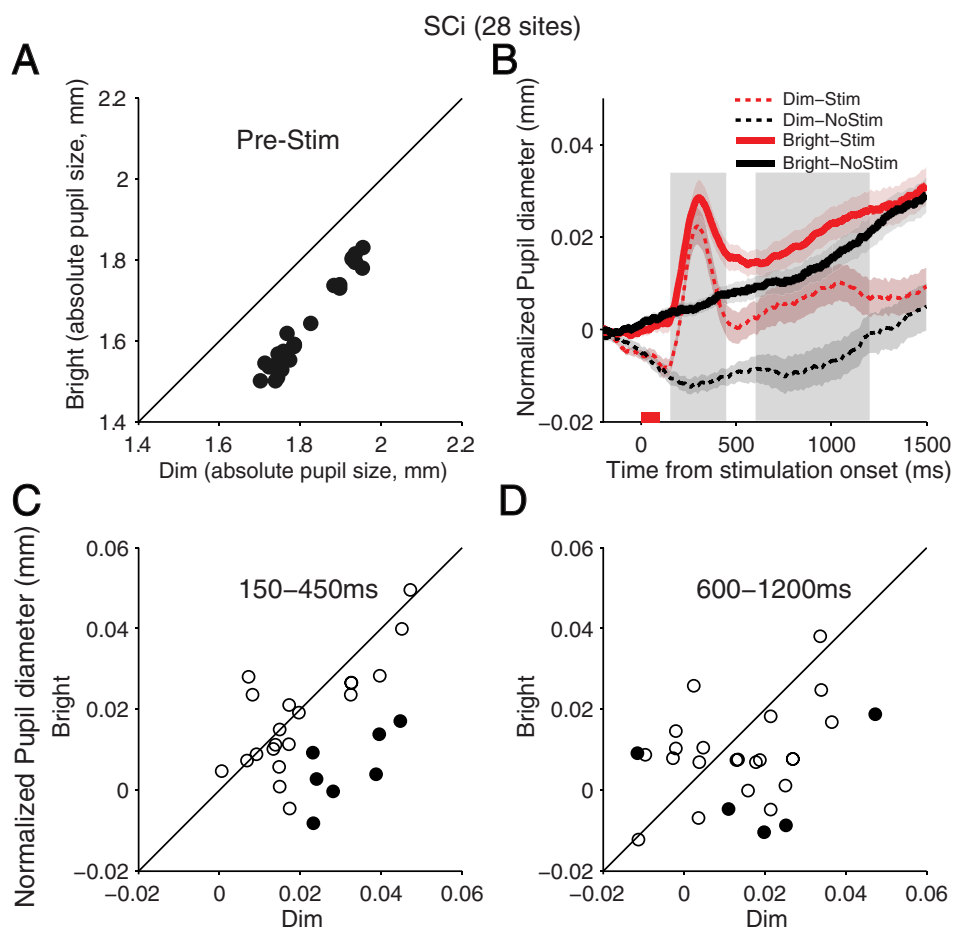

D

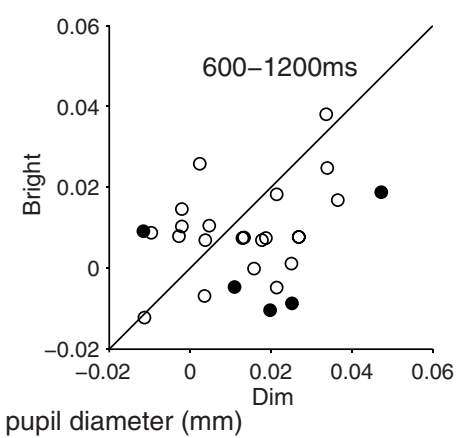

Figure 3. Comparison of the magnitude of pupil dilation evoked by SCi microstimulation for two levels of background illumination across all stimulation sites. $A$, The absolute pupil values before microstimulation between the dim and bright backgrounds. Filled circles indicate differences that are statistically significant $(p<0.05)$. B, Pupil dynamics of SCi microstimulation. Red and black lines indicate the microstimulation and no-stimulation trials, respectively, and the dashed and solid lines indicate the dim and bright backgrounds, respectively. Red bar indicates the time line of microstimulation. The phasic epoch ( $150-450 \mathrm{~ms}$ after the stimulation onset) and the sustained epoch ( $600-1200 \mathrm{~ms}$ after the stimulation onset) are shaded in gray. The shaded regions and pupillary response traces represent \pm 1 SEM. C, D, The pupil dilation values of the SCi microstimulation between the dim and the bright conditions during the phasic $(\boldsymbol{C})$ and the sustained $(\boldsymbol{D})$ epochs. Filled circles indicate differences that are statistically significant $(p<0.05)$. Stim, Stimulation; NoStim, no stimulation.

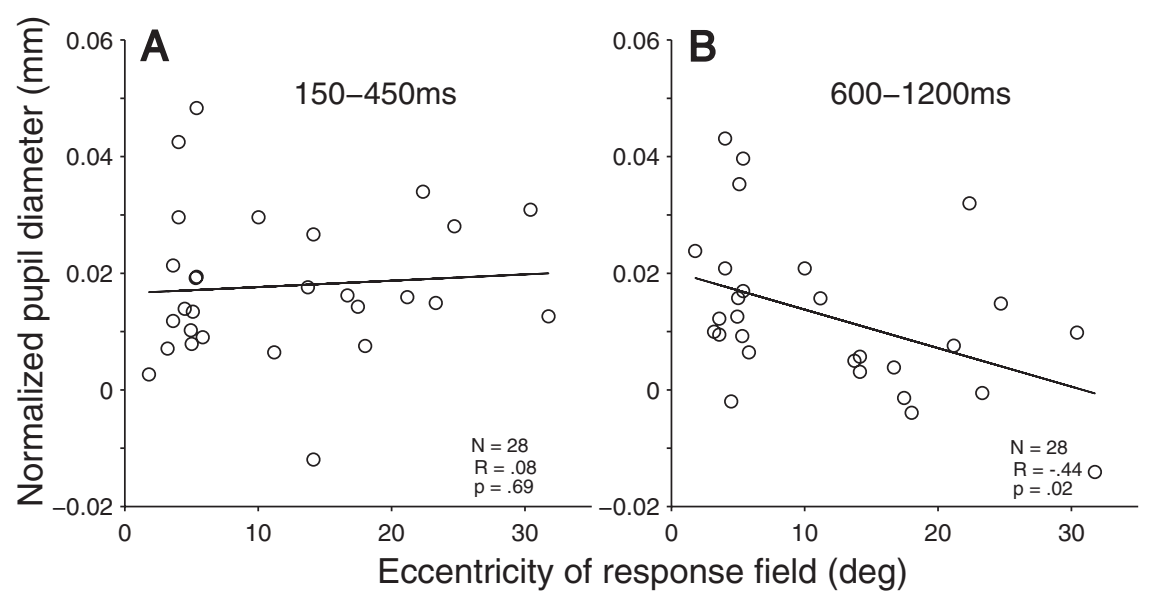

Figure 4. Effects of $S C$ i stimulation as a function of the eccentricity of stimulation site. $A, B$, Correlation between the eccentricity of stimulation site and the size of pupil dilation during the phasic $(\boldsymbol{A})$ and sustained $(\boldsymbol{B})$ epochs. Black lines indicate the regression line.

\section{Distinct functional roles for SC layers}

That stimulation of the $\mathrm{SCi}$, but not the SCs, evoked transiently pupil dilation may suggest distinct roles for the SC sublayers in the orienting reflex. The functional differences between the SC layers on the orienting reflex beyond saccadic eye movements have received little study, although their anatomical distinctions have been intensively explored (White and Munoz, 2011). The
$\mathrm{SC}$ receives afferent projections from visual areas exclusively and is hypothesized to represent a visual salience map (Boehnke and Munoz, 2008). The SCi, however, receives not only SC inputs, but also signals from many cortical and subcortical areas, and sends motor commands to the brainstem premotor circuitry to execute orienting responses (Munoz et al., 1991; Rodgers et al., 2006). Because the SCi integrates sensory-related signals from visual areas and goal-directed signals from cortical and subcortical areas, it is hypothesized to represent a priority map (Fecteau and Munoz, 2006; Boehnke and Munoz, 2008). However, studies exploring SC microstimulation on the shift of attention only stimulated the SCi (Kustov and Robinson, 1996; Cavanaugh and Wurtz, 2004) or reported results including one SC microstimulation site (Müller et al., 2005). An earlier study of SC stimulation on pupil dilation in one anesthetized monkey did not report which layer was stimulated (Jampel, 1960). Nevertheless, one study using single-unit recording has shown that only $\mathrm{SCi}$ neurons were modulated by covert shifts of attention (Ignashchenkova et al., 2004). Together, the close relationship between the SCi and various components of the orienting reflex suggests that the orienting reflex is not only driven by bottom-up inputs, but also modulated by top-down factors.

\section{Possible functions of pupil dilation}

The function of orienting reactions is thought to increase the sensitivity of sense organs to efficiently discern the event and mobilize the body for whatever action is required (Lynn, 1966). The advantages of shifts of gaze and attention are apparent, because saccadic eye movements foveate a target of interest to provide the high visual resolution and covert attention enhances the neuronal responses to selected targets throughout the cortex (for recent review, see Reynolds and Chelazzi, 2004; Baluch and Itti, 2011; Carrasco, 2011). However, the advantage of pupil dilation is less obvious. Although it has been explicitly proposed that pupil dilation serves to enhance visual sensitivity (Lynn, 1966), there is little empirical evidence to support the argument (Nieuwenhuis et al., 2011). An increase in pupil size can enhance visual sensitivity; however, this comes with a reduction in image sharpness caused by aberrations (Laughlin, 1992). Therefore, instead of constant dilating, the natural pupil size varies according to background illumination. The modulation is thought to regulate the tradeoff between sensitivity and sharpness for the optimization of image quality (Leibowitz, 1952; Campbell and Gregory, 1960; Laughlin, 1992). Consis- 
A

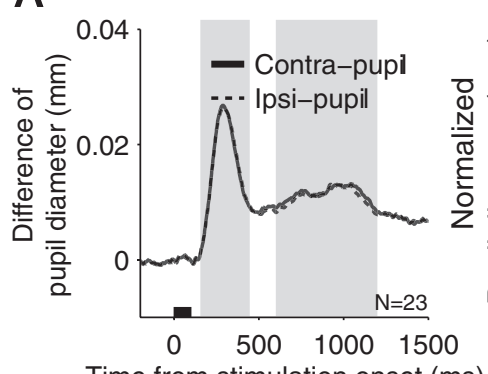

Time from stimulation onset (ms)

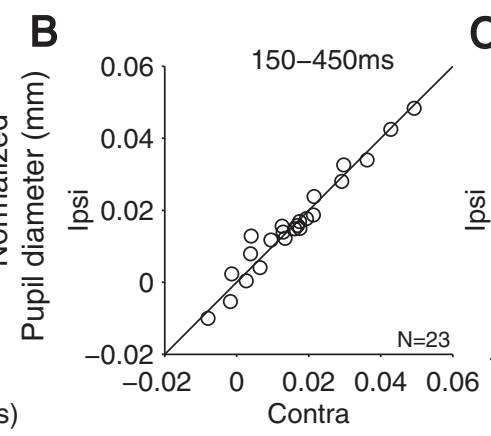

Normalized pupil diameter (mm)

Figure 5. Summary of SCi microstimulation effects on the contralateral or ipsilateral pupil of the stimulation site $(n=23)$. $\boldsymbol{A}$, Pupil dynamics of stimulation minus no-stimulation conditions. Black bar indicates the time line of microstimulation, and the $150-450$ and $600-1200$ ms epochs are shaded in gray. $B$, C, Pupil dilation values between contra and ipsi of the stimulation site on the phasic $(\boldsymbol{B})$ and sustained (C) epochs. Contra, contralateral; Ipsi, ipsilateral.

tent with this, the optimal pupil size for highest acuity was found to be close to the size of the natural pupil under different levels of luminance (Woodhouse, 1975).

If the stimulation-evoked pupil dilation is functionally related to visual sensitivity, then the size of pupil dilation should be restrained such that the enlarged pupil size can enhance visual sensitivity without exceeding the natural pupil size required for optimal visual acuity. Furthermore, because the natural pupil size is larger under lower illuminance values, the size of pupil dilation should be larger in the dimmer condition. That stimulation-evoked pupil dilation was small in magnitude (Fig. 2A) and was greater under dimmer illuminance (Fig. $3 C, D$ ) supports the idea that pupil dilation evoked by SCi microstimulation may be associated with increased visual sensitivity.

Given this small magnitude of change, does this stimulusdriven pupil dilation have a functional use? We have shown previously that pupil size at the onset of target presentation is negatively correlated with saccadic reaction times in various visual/oculomotor tasks (Wang et al., 2011). So, a slightly larger pupil during target onset can shorten the latency to orient, perhaps contributing to the probability with which an organism catches its prey or effectively flees from a predator. Because our results provide only indirect evidence regarding the functions of pupil dilation on enhancing visual sensitivity, it requires future investigation to support the hypothesis.

\section{Coordination between pupil dilation and saccadic eye movements}

Pupil dilation differs from saccadic eye movements in a number of ways. Saccadic eye movements are very quick, and latencies to evoke saccades with SCi microstimulation can be as short as 30 ms (Robinson, 1972). In contrast, the pupil response takes longer to initiate and lasts longer: SCi stimulation-evoked pupil responses are typically initiated within $150 \mathrm{~ms}$ and are sustained for $>1$ s. Moreover, unlike saccadic eye movements, the pupil cannot enhance visual processing in a spatially specific manner. How are they coordinated to optimize performance? Although it remains unclear how the coordination between various components of the orienting reflex is achieved, we think pupil dilation can work with saccadic eye movements to optimize performances under two scenarios. When the salient stimulus cannot be spatially located (e.g., sounds), pupil dilation can possibly increase visual sensitivity to detect possible target locations. When the salient stimulus is spatially localized, sac- cadic eye movements are often initiated to foveate the target before pupil dilation has even started. In this instance, dilated pupils will enhance signal processing of the target and its surrounding locations immediately after foveation. In both cases, pupil dilation may coordinate with saccadic eye movements to prepare appropriate actions.

\section{Anatomical pathways supporting pupil dilation}

Although orienting responses are mediated by the SC (Boehnke and Munoz, 2008), pupil dilation differs from other components of the orienting reflex in a number of ways. Pupil size is controlled by complex interactions between the dilation and the constriction pathways (Loewenfeld, 1999). SC neurons project ipsilaterally to the pretectal olivary nucleus (Harting et al., 1980), and neurons in this nucleus project bilaterally to the Edinger-Westphal (EW) nucleus, which contains the parasympathetic, preganglionic neurons that control pupil constriction (Gamlin, 2006). Because microstimulation of the SCs did not elicit pupil dilation, the pathways that mediate it are unlikely via SC efferent projections. The SCi projects directly to the EW nucleus (Harting et al., 1980), and a subset of output neurons in the SCi (X neurons) are possibly involved in the projection (Moschovakis et al., 1988a). This pathway can dilate the pupil indirectly by inhibiting the pupil constriction pathways. Efferent projections of the SCi to the mesencephalic cuneiform nucleus (CNF) are via the ipsilateral tectopontine-tectobulbar tract (Harting, 1977; Huerta and Harting, 1984; May, 2006), which conveys collicular motor commands, and this structure regulates stress-related and defensive responses (Dean et al., 1989; Korte et al., 1992). T neurons of the SCi possibly mediate the anatomical connection from the SCi to the CNF nucleus (Moschovakis et al., 1988a). Stimulation of the CNF activates sympathetic vasomotor outflow (Verberne, 1995), and the dilator muscle of the pupils is regulated by the sympathetic pathway (Loewenfeld, 1999). Moreover, the CNF projects ipsilaterally to the locus ceruleus where it also connects to the EW nucleus (Korte et al., 1992). We propose that projections from the SCi to the EW nucleus and the CNF may underlie the stimulation-evoked pupil dilation observed in the current study, by activating the sympathetic pathway and inhibiting the parasympathetic pathway.

\section{Links to cognitive processing}

Pupil dilation has been an effective indicator for cognitive processing (Beatty, 1982), and it is associated with processes such as 
target detection (Privitera et al., 2010), covert orienting (Gabay et al., 2011), and subjective perception (Einhauser et al., 2008). The neural locus that mediates between cognitive states and pupil dilation is less understood, although the locus ceruleus-norepinephrine system is regularly assumed (Aston-Jones and Cohen, 2005). Recently, the SC has been suggested to be involved causally on covert spatial selection of goal-related signals for a perceptual judgment (Lovejoy and Krauzlis, 2010). The covert selection of relevant information is required regularly to perform cognitive tasks successfully. The modulation of pupil dynamics by SC microstimulation has the potential to entail another neural substrate to explain the close relationship between pupil dilation and various cognitive processing.

\section{References}

Aston-Jones G, Cohen JD (2005) An integrative theory of locus coeruleusnorepinephrine function: adaptive gain and optimal performance. Annu Rev Neurosci 28:403-450.

Bala AD, Takahashi TT (2000) Pupillary dilation response as an indicator of auditory discrimination in the barn owl. J Comp Physiol A 186:425-434

Baluch F, Itti L (2011) Mechanisms of top-down attention. Trends Neurosci $34: 210-224$

Beatty J (1982) Task-evoked pupillary responses, processing load, and the structure of processing resources. Psychol Bull 91:276-292.

Boehnke SE, Munoz DP (2008) On the importance of the transient visual response in the superior colliculus. Curr Opin Neurobiol 18:544-551.

Campbell FW, Gregory AH (1960) Effect of size of pupil on visual acuity. Nature 187:1121-1123.

Carrasco M (2011) Visual attention: the past 25 years. Vision Res $51: 1484-1525$.

Cavanaugh J, Wurtz RH (2004) Subcortical modulation of attention counters change blindness. J Neurosci 24:11236-11243.

Cynader M, Berman N (1972) Receptive-field organization of monkey superior colliculus. J Neurophysiol 35:187-201.

Dean P, Redgrave P, Westby GW (1989) Event or emergency? Two response systems in the mammalian superior colliculus. Trends Neurosci 12:137-147.

Einhauser W, Stout J, Koch C, Carter O (2008) Pupil dilation reflects perceptual selection and predicts subsequent stability in perceptual rivalry. Proc Natl Acad Sci U S A 105:1704-1709.

Fecteau JH, Munoz DP (2006) Salience, relevance, and firing: a priority map for target selection. Trends Cogn Sci 10:382-390.

Gabay S, Pertzov Y, Henik A (2011) Orienting of attention, pupil size, and the norepinephrine system. Atten Percept Psychophys 73:123-129.

Gamlin PD (2006) The pretectum: connections and oculomotor-related roles. Prog Brain Res 151:379-405.

Goldberg ME, Wurtz RH (1972) Activity of superior colliculus in behaving monkey. I. Visual receptive fields of single neurons. J Neurophysiol 35:542-559.

Hall WC, Moschovakis A (2003) The superior colliculus: new approaches for studying sensorimotor integration. Boca Raton, FL: CRC.

Harting JK (1977) Descending pathways from the superior collicullus: an autoradiographic analysis in the rhesus monkey (Macaca mulatta). J Comp Neurol 173:583-612.

Harting JK, Huerta MF, Frankfurter AJ, Strominger NL, Royce GJ (1980) Ascending pathways from the monkey superior colliculus: an autoradiographic analysis. J Comp Neurol 192:853-882.

Hays AV, Richmond BJ, Optican LM (1982) A UNIX-based multipleprocess system for real-time data acquisition and control. In: WESCON Conference Proceedings, pp 1-10.

Hess WR, Buergi S, Bucher V (1946) Motorische funktionen des tektal-und tegmentalgebietes (motor functions of tectal and tegmental areas). Monatsschr Psychiatr Neurol 112:1-52.

Hikosaka O, Wurtz RH (1983) Effects on eye movements of a GABA agonist and antagonist injected into monkey superior colliculus. Brain Res $272: 368-372$.

Huerta M, Harting J (1984) Connectional organization of the superior colliculus. Trends Neurosci 7:286-289.

Ignashchenkova A, Dicke PW, Haarmeier T, Thier P (2004) Neuron- specific contribution of the superior colliculus to overt and covert shifts of attention. Nat Neurosci 7:56-64.

Jampel RS (1960) Convergence, divergence, pupillary reactions and accommodation of the eyes from faradic stimulation of the macaque brain. J Comp Neurol 115:371-399.

Korte SM, Jaarsma D, Luiten PG, Bohus B (1992) Mesencephalic cuneiform nucleus and its ascending and descending projections serve stress-related cardiovascular responses in the rat. J Auton Nerv Syst 41:157-176.

Kustov AA, Robinson DL (1996) Shared neural control of attentional shifts and eye movements. Nature 384:74-77.

Laughlin SB (1992) Retinal information capacity and the function of the pupil. Ophthalmic Physiol Opt 12:161-164.

Leibowitz H (1952) The effect of pupil size on visual acuity for photometrically equated test fields at various levels of luminance. J Opt Soc Am 42:416-422.

Loewenfeld IE (1999) The pupil: anatomy, physiology, and clinical applications. Boston: Butterworth-Heinemann.

Lovejoy LP, Krauzlis RJ (2010) Inactivation of primate superior colliculus impairs covert selection of signals for perceptual judgments. Nat Neurosci 13:261-266

Lynn R (1966) Attention, arousal and the orientation reaction. Oxford, UK: Pergamon.

Marino RA, Rodgers CK, Levy R, Munoz DP (2008) Spatial relationships of visuomotor transformations in the superior colliculus map. J Neurophysiol 100:2564-2576.

May PJ (2006) The mammalian superior colliculus: laminar structure and connections. Prog Brain Res 151:321-378.

Mohler CW, Wurtz RH (1977) Role of striate cortex and superior colliculus in visual guidance of saccadic eye movements in monkeys. J Neurophysiol 40:74-94.

Moresi S, Adam JJ, Rijcken J, Van Gerven PW (2008) Cue validity effects in response preparation: a pupillometric study. Brain Res 1196:94-102.

Moschovakis AK, Karabelas AB, Highstein SM (1988a) Structure-function relationships in the primate superior colliculus. I. Morphological classification of efferent neurons. J Neurophysiol 60:232-262.

Moschovakis AK, Karabelas AB, Highstein SM (1988b) Structure-function relationships in the primate superior colliculus. II. Morphological identity of presaccadic neurons. J Neurophysiol 60:263-302.

Müller JR, Philiastides MG, Newsome WT (2005) Microstimulation of the superior colliculus focuses attention without moving the eyes. Proc Natl Acad Sci U S A 102:524-529.

Munoz DP, Guitton D, Pélisson D (1991) Control of orienting gaze shifts by the tectoreticulospinal system in the head-free cat. III. Spatiotemporal characteristics of phasic motor discharges. J Neurophysiol 66:1642-1666.

Netser S, Ohayon S, Gutfreund Y (2010) Multiple manifestations of microstimulation in the optic tectum: eye movements, pupil dilations, and sensory priming. J Neurophysiol 104:108-118.

Nieuwenhuis S, de Geus EJ, Aston-Jones G (2011) The anatomical and functional relationship between the $\mathrm{P} 3$ and autonomic components of the orienting response. Psychophysiology 48:162-175.

Privitera CM, Renninger LW, Carney T, Klein S, Aguilar M (2010) Pupil dilation during visual target detection. J Vis 10:3.

Reynolds JH, Chelazzi L (2004) Attentional modulation of visual processing. Annu Rev Neurosci 27:611-647.

Robinson DA (1963) A method of measuring eye movement using a scleral search coil in a magnetic field. IEEE Trans Biomed Eng 10:137-145.

Robinson DA (1972) Eye movements evoked by collicular stimulation in the alert monkey. Vision Res 12:1795-1808.

Rodgers CK, Munoz DP, Scott SH, Paré M (2006) Discharge properties of monkey tectoreticular neurons. J Neurophysiol 95:3502-3511.

Schiller PH, Koerner F (1971) Discharge characteristics of single units in superior colliculus of the alert rhesus monkey. J Neurophysiol 34:920-936.

Schiller PH, Sandell JH, Maunsell JH (1987) The effect of frontal eye field and superior colliculus lesions on saccadic latencies in the rhesus monkey. J Neurophysiol 57:1033-1049.

Sokolov EN (1963a) Higher nervous functions; the orienting reflex. Annu Rev Physiol 25:545-580.

Sokolov EN (1963b) Perception and the conditioned reflex. New York: Macmillan.

Sparks DL (1978) Functional properties of neurons in the monkey superior 
colliculus: coupling of neuronal activity and saccade onset. Brain Res $156: 1-16$.

Sparks DL (1986) Translation of sensory signals into commands for control of saccadic eye movements: role of primate superior colliculus. Physiol Rev 66:118-171.

Stelmack RM, Siddle DA (1982) Pupillary dilation as an index of the orienting reflex. Psychophysiology 19:706-708.

Van Gisbergen JA, Van Opstal AJ, Tax AA (1987) Collicular ensemble coding of saccades based on vector summation. Neuroscience 21:541-555.

Verberne AJ (1995) Cuneiform nucleus stimulation produces activation of medullary sympathoexcitatory neurons in rats. Am J Physiol 268:R752-R758.
Wang C-A, Boehnke SE, White BJ, Munoz DP (2011) Subthreshold microstimulation of the superior colliculus induces pupil dilation. J Vis 11:189.

White BJ, Boehnke SE, Marino RA, Itti L, Munoz DP (2009) Colorrelated signals in the primate superior colliculus. J Neurosci 29: 12159-12166.

White BJ, Munoz DP (2011) The superior colliculus. In: Oxford handbook of eye movements (Liversedge S, Gilchrist I, Everling S, eds), Oxford UP: Oxford, UK.

Woodhouse JM (1975) The effect of pupil size on grating detection at various contrast levels. Vision Res 15:645-648.

Wurtz RH, Goldberg ME (1971) Superior colliculus cell responses related to eye movements in awake monkeys. Science 171:82-84. 